\title{
Management of Invasive Cervical Resorption Involving the Pulp by Surgical and Restorative Therapy
}

\author{
${ }^{1}$ Vinisha Ranna, ${ }^{2}$ Abrar Sayed
}

\begin{abstract}
Invasive cervical resorption (ICR) is a form of external resorption which affects the cervical area of permanent teeth and may progress to involve the pulp. It can cause insidious loss of tooth structure, which often is undetected until late in the process. Two cases, both involving the maxillary left central incisor where cone-beam computed tomography (CBCT) revealed an invasive cervical resorptive lesion, extending from the cervical to mid root region and perforating the pulp chamber. The cases were managed surgically with the help of a dental operating microscope (DOM). Nine months follow-up revealed resolution of the lesion and restoration of the affected teeth to functional occlusion. This report emphasizes the restorability of advanced lesions of $I C R$, if adequate diagnostic and treatment equipment is used.
\end{abstract}

Keywords: Cone-beam computed tomography, Invasive cervical resorption involving the pulp, Mineral trioxide aggregate.

How to cite this article: Ranna V, Sayed A. Management of Invasive Cervical Resorption Involving the Pulp by Surgical and Restorative Therapy. Int J Experiment Dent Sci 2015;4(2): 130-133.

Source of support: Nil

Conflict of interest: None

\section{INTRODUCTION}

Traditionally as well as in the light of current knowledge, teeth with invasive cervical resorption ${ }^{1}$ involving the pulp (Heithersays class 4 lesion) ${ }^{2}$ are condemned to extraction due to its suggested poor prognosis. ${ }^{3}$ Possible etiological agents are periodontal disease, surgical procedures, trauma, orthodontic movement and endogenous bleaching. ${ }^{4}$

Invasive cervical resorption begins as an inflammation of periodontal tissue which progresses to shallow cavities in the cementum. The process extends into the

\footnotetext{
${ }^{1}$ Intern, ${ }^{2}$ Associate Professor

${ }^{1,2}$ Department of Conservative Dentistry and Endodontics Government Dental College and Hospital, Mumbai Maharashtra, India

Corresponding Author: Vinisha Ranna, Intern, Department of Conservative Dentistry and Endodontics, Government Dental College and Hospital, Mumbai, Maharashtra, India, Phone: 9920989792, e-mail: vinisha.ranna@gmail.com
}

dentin and if it remains undetected, as is often the case, pulp involvement may occur.

Surgical and nonsurgical management has been suggested for the treatment of ICR. The surgical treatment of ICR has generally involved periodontal flap reflection, curettage and restoration of the defect with materials, such as composite resin, glass ionomer cement (GIC), resin-modified GIC or mineral trioxide aggregate (MTA).

Restoration of ICR defects with the combination of different restorative materials to attain optimum mechanical and biological properties has been described only in one case where microfilled composite and resin-modified GIC were used in a technique coined-'reverse sandwich restoration. ${ }^{5}$

In the following two clinical cases such teeth have been salvaged through conservative management by performing surgical curettage of the lesion and restoration of the defect with different materials.

\section{CASE REPORTS}

\section{Case 1}

A 38-year-old male having severe abrasion of \#8 and \#9 (Fig. 1A) with pulp exposure which were nontender to percussion reported to the department of conservative dentistry and endodontics. The teeth did not demonstrate tenderness or pathological mobility. Tooth number appeared to have a deep cervical defect, which appeared resorptive in nature.

Radiographic examination revealed a periapical radiolucency with \#8. With \#9, there appeared to be a large radiolucency extending from cervical to mid root level (Fig. 1B). Cone-beam computed tomography (CBCT) revealed a resorption defect perforating the pulp chamber with \#9 (Figs 1C to E).

The diagnosis of ICR, Heithersays class 4 lesion was formulated for \#9. Endodontic treatment was planned for \#8 and \#9 followed by curettage of lesions and then restoration for a biocompatible seal. A surgical approach was planned to treat \#9. After local infiltration with $2 \%$ lignocaine with adrenaline 1:80,000 (ICPA health products Ltd.), a flap was raised.

Entire treatment was performed using a dental operating microscope (DOM) (Carl Zeiss OPMI). With \#9 
the resorptive defect clinically extended from the labial to the distal root surface.

The root canal could not be located during access opening due to profuse bleeding from the tissue in the defect (Fig. 1F). The soft tissue filling the defect was curetted. This gave a clear access to the root canal and cleaning and shaping was completed. The canal was obturated with gutta percha and zinc oxide eugenol sealer (Shiva products) till the defect. Light cure resin-based composite (Matrix micro hybrid composite, europe) was used to restore normal root contour (Fig. 1G). Following manufacturer specifications, MTA was placed (MTA plus, Prevest Denpro Limited ) over the composite (Fig. 1H).

The flap was sutured back, and a noneugenol periodontal pack was placed. One week recall for pack removal revealed normal tissue healing (Figs 1I and J).

\section{Case 2}

A 49-year-old male patient presented with the chief complaint of food lodgment and pain with \#9 (Fig. 2A). On probing the tooth revealed a cervical subgingival defect. The tooth was firm and nontender.

Radiographs revealed an extensive defect on cervical area of the root surface crossing to the pulp (Fig. 2B). Conebeam computed tomography revealed a large resorptive defect, involving the pulp uptil mid root level (Figs 2C to E). A diagnosis of ICR, class 4 lesion was formulated.

Surgical management as in the previous case was done, restoring the defect with MTA (Figs 2F and G). The surface of the MTA was covered with freeze dried irradiated bone graft (Tata Memorial Center) over which was placed collagen resorbable membrane (Tata Memorial Center, Tissue bank) (Fig. 2H). Following closure with 3.0 silk, routine postoperative care was instituted. Patient was recalled in a week for suture removal and follow-up (Figs 2I and J).

Nine months follow-up in both cases did not reveal any recurrence of resorption, gingival healing appeared to be complete with normal probing depths and no gingival recession (Figs 3 and 4). There was no periodontal pocket or other pathological signs in relation to the restoration.
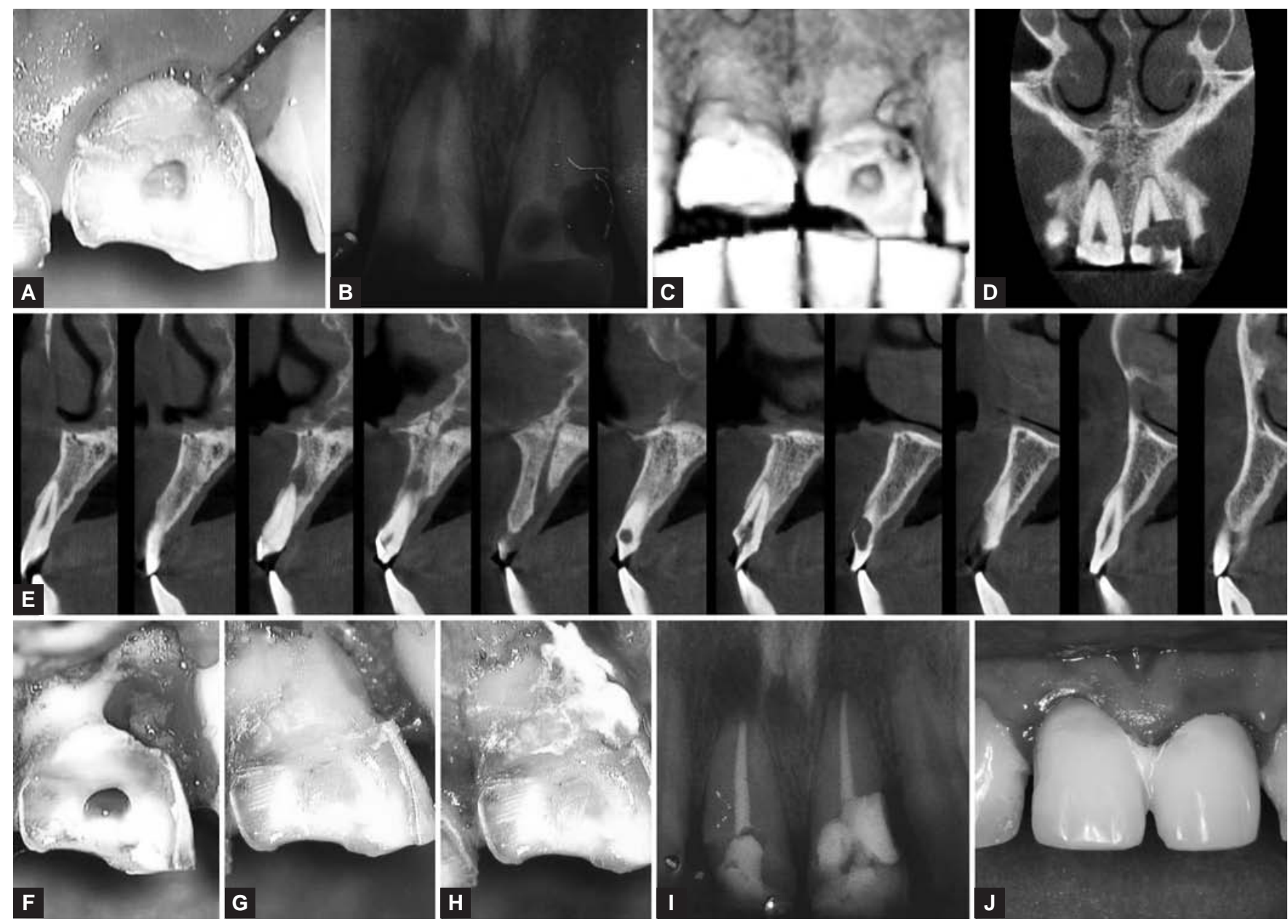

Figs 1A to J: (A) Preoperative image showing severe abrasion of the central incisors, (B) preoperative radiograph, (C) 3D reconstruction on CBCT, (D) extension of defect in coronal section on CBCT, (E) extension of the defect in sagittal sections, $(F)$ intraoperative view of the defect, granulation tissue seen, $(G)$ composite build up with red filter in place, $(H)$ MTA placed above composite (as seen under red filter), (I) postoperative radiograph and $(\mathrm{J})$ postoperative restoration 

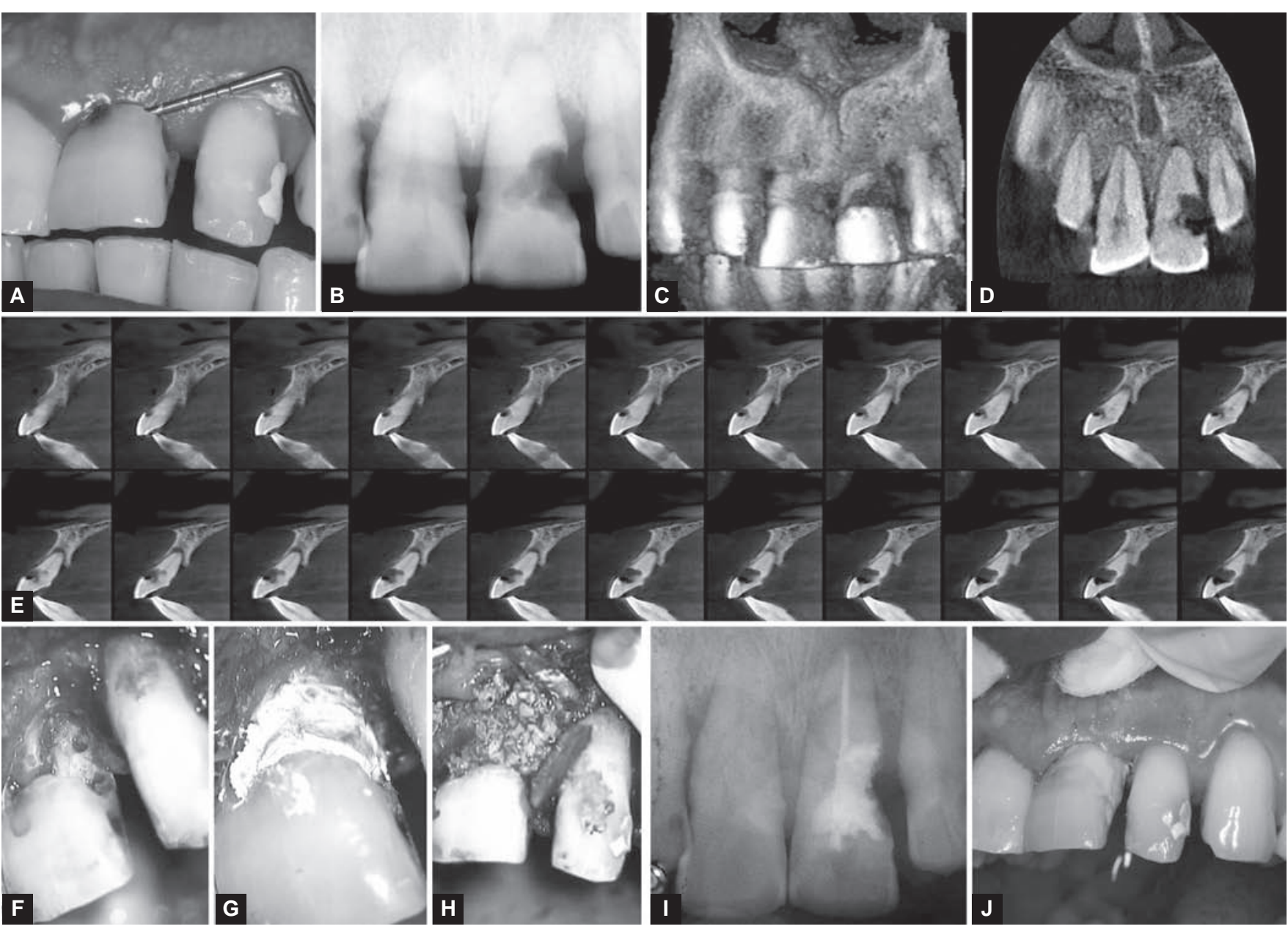

Figs 2A to J: (A) Preoperative clinical presentation, (B) preoperative radiograph, (C) 3D reconstruction of the defect as seen on CBCT, (D) extension of defect in coronal section on CBCT, (E) extension of the defect in sagittal sections, (F) intraoperative view of the defect, (G) mineral trioxide aggregate layer in place, $(\mathrm{H})$ bone graft with membrane placed over the MTA, (I) postoperative radiograph and $(\mathrm{J})$ postoperative restoration
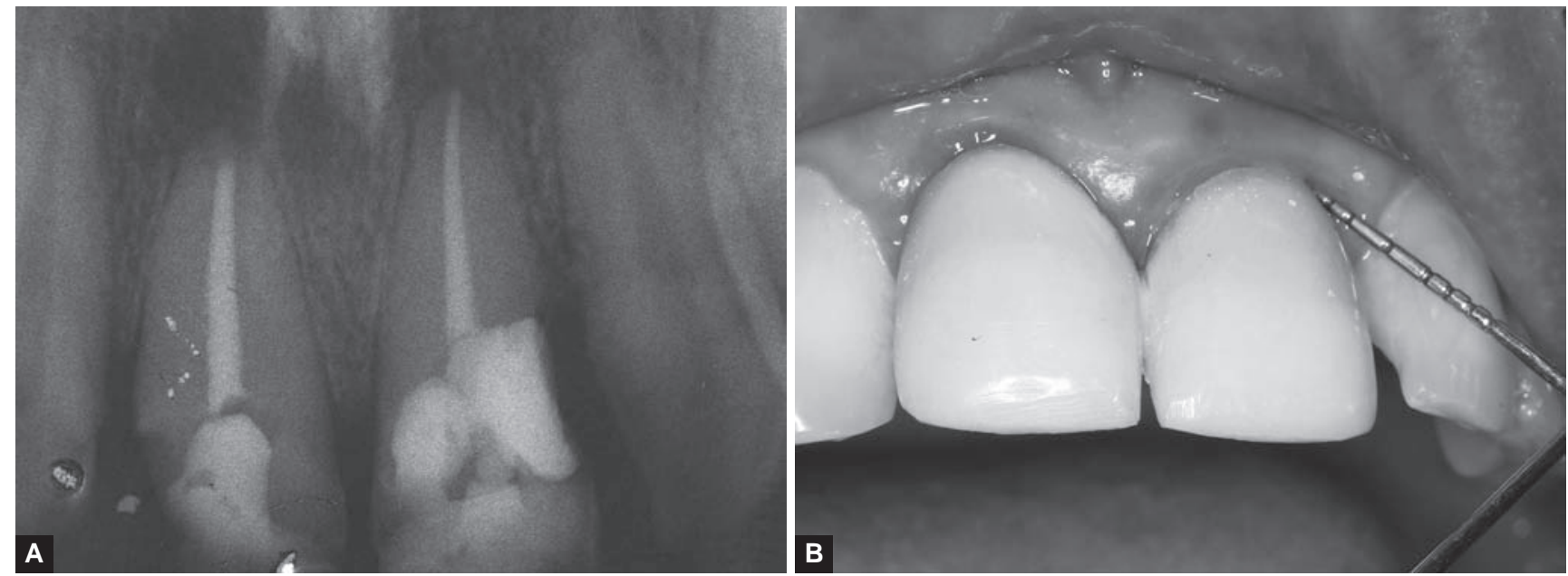

Figs 3 A and B: Nine months follow-up with restoration intact and adequate healing in case 1

\section{DISCUSSION}

Keeping in view the expanded gamut of clinical equipment, materials and techniques, we have attempted to review the basic clinical protocol of management of such cases.
Heithersay who first classified ICR suggested surgical management of ICR lesions involving the pulp. ${ }^{3} \mathrm{He}$ suggested that although prosthodontic replacement may be necessary in most cases, an attempt can be made to restore such teeth to function if the bone loss can be repaired. 

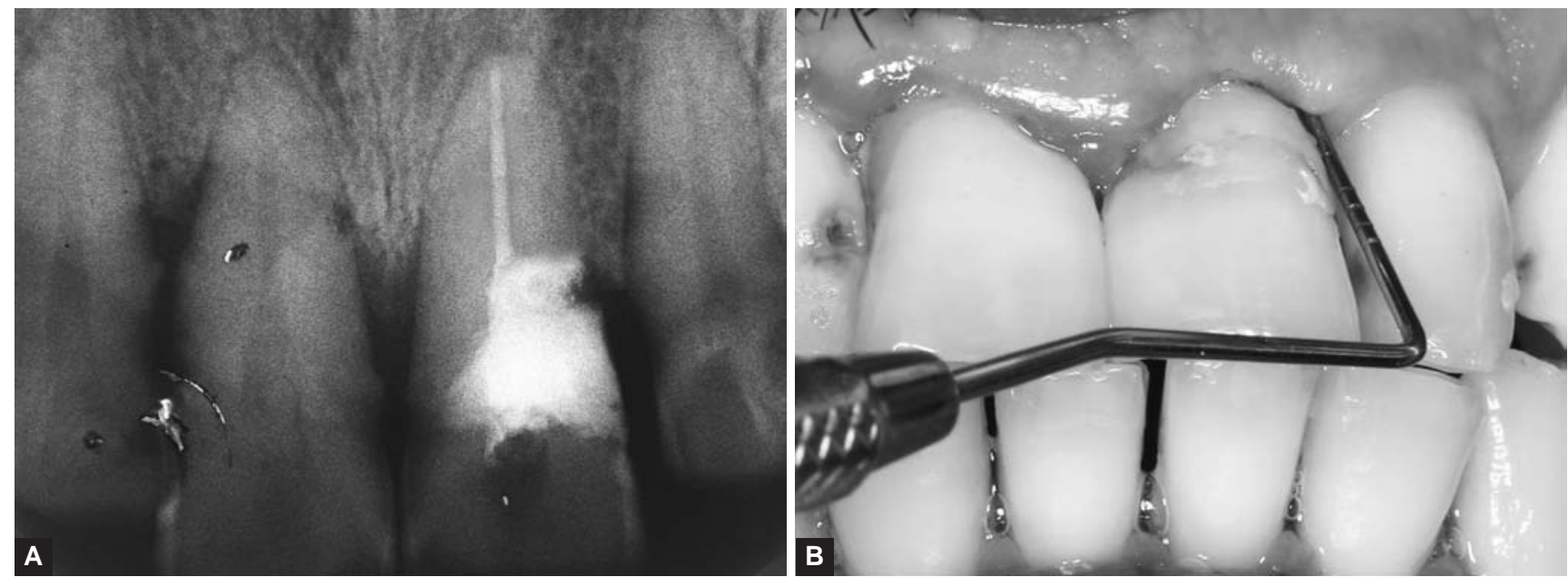

Figs 4A and B: Nine months follow-up with restoration intact and adequate healing in case 2

In these cases, both the lesions treated were subgingival and supraosseous. Cone-beam computed tomography and microsurgery were used to clinically judge the extent of the lesion. They proved to be of invaluable help in locating the defect, curettage, restoration and final closure of the wound. After the curettage in the first case, the lost tooth structure was restored with a bonded resin restoration with the intention of strengthening the tooth as the defect was very extensive. Mineral trioxide aggregate $^{6,7}$ was laid on the surface to make the environment biologically conducive to osseous healing. 8,9

The second case had a very similar lesion. Though it was less extensive, it had considerable vertical bone loss associated with the resorptive lesion. So, it was decided to repair the defect with MTA followed by placement of collagen membrane and bone grafting material to get good periodontal and osseous repair or regeneration. Nine months follow-up showed excellent endodontic and periodontal healing.

\section{CONCLUSION}

This technique took a conservative approach to management of ICR defects involving the pulp. In these cases, restoration of the defect and $>5 \mathrm{~mm}$ attachment gain was achieved at nine months follow-up. The dental examination should be timed appropriately to allow any necessary dental treatment to be carried out in a planned manner. ${ }^{10}$

\section{Clinical Significance}

Careful case selection, and informing the patient of the questionable clinical outcome in such cases is of impor- tance. Judicious use of advanced diagnostic equipment is advantageous to formulate a treatment plan. Further investigations and studies are warranted to develop exact protocols for management and assertion of treatment outcomes.

\section{REFERENCES}

1. Heithersay GS. Invasive cervical resorption: an analysis of potential predisposing factors. Quintessence Int 1999;30(2): 83-95.

2. Heithersay GS. Management of tooth resorption. Aust Dental J 2007;52(Suppl 1):105-121.

3. Heithersay GS. Invasive cervical resorption. Endod Topic 2004;7(Suppl 1):73-92.

4. Augusto J. Invasive cervical resorption: etiology, diagnosis, classification and treatment. J Contemp Dent Pract 2012 Sep; 13(5):723-728.

5. Thilla SV, Ramchandran T, Deivanayagam K. Reverse sandwich restoration for the management of invasive cervical resorption: a case report. J Endod 2011;37(5):706-710.

6. Silveira LM, Silveira CF, Martos J, Piovesan EM, César Neto JB. Clinical technique for invasive cervical root resorption. J Conserv Dentist 2001;14(4):440-444.

7. Baek SH, Plenk H, Kim S. Periapical tissue responses and cementum regeneration with amalgam, Super EBA and MTA as root-end filling materials. J Endod 2005;31(6):444-449.

8. Parirokh M, Torabinejad M. Mineral trioxide aggregate: a comprehensive literature review-part I: chemical, physical and antibacterial properties. J Endod 2010;36(3):16-27.

9. Zhang H, Pappen FG, Haapasalo M. Dentin enhances the antibacterial effect of mineral trioxide aggregate and bioaggregate. J Endod 2009;35(2):221-224.

10. Saini R, Sugandha, Saini S. The importance of oral health in kidney disease. Saudi J Kidney Dis Transplant 2010;21(6): 1151-1152. 